8 Peterson, P. A., Cunningham, B. A., Berggård, I. and Edelman, G. M., Proc. Natl. Acad. Sci. USA 1972. 69: 1697.

9 Smithies, O. and Poulik, M. D., Science 1972. 175: 187.

10 Hahn, G. S. and Hamburger, R. N., J. Immunol. 1981. 126: 459.

11 Tragardh, L., Rask, L., Wiman, K., Fohlman, J. and Peterson, P. A., Proc. Natl. Acad. Sci. USA 1980. 77: 1129.

12 Cotmore, S. F., Crowhurst, S. A. and Waterfield, M. D., Eur. J. Immunol. 1981. 11: 597.

13 McKenzie, J. L., Allen, A. K. and Fabre, J. W., Biochem. J. 1981. 197: 629

14 McKenzie, J. L. and Fabre, J. W., Transplantation 1981. 31: 275.

15 Rostas, J. A. P., Shevenan, T. A., Sinclair, C. M. and Jeffrey, P. L., Biochem. J. 1983. 213: 143.

16 Mansour, M. H. and Cooper, E. L., J. Immunol. 1984. 132: 2515.

17 Berrill, N. J., Oxford University Press, New York 1955.

18 Endean, R., Q. J. Microsc. Sci. 1960. 101: 177.

19 Overton, J., J. Morphol. 1966. 119: 305.

20 Reddy, A. L., Bryan, B. and Hildemann, W. H., Immunogenetics 1975. I: 584

21 Oka, H. and Watanabe, H., Proc. Japan Acad. 1957. 33: 657.

22 Scofield, V. L., Schlumpberger, J. M., West, L. A. and Weissman, I. L., Nature 1982. 295: 499 .

23 Warr, G. W., Vasta, G. R., Ledford, B. E. and Marchalonis, J. J., J. Immunogen. 1983. 10: 17.

24 Vasta, G. R. and Marchalonis, J. J., in Bøg-Hansen, T. C. (Ed.), Lectins: Biology, Biochemistry and Clinical Biochemistry, W. de Gryter, Berlin 1983, p. 46.

25 Warr, G. W., Decker, J. M., Mandel, T. W., DeLuca, D., Hudson, R. and Marchalonis, J. J., Aust. J. Exp. Biol. Med. Sci. 1977. 55: 151 .
26 Wright, R. K. and Cooper, E. L., Am. Zool. 1981. 21: 974.

27 Wright, R. K. and Cooper, E. L., in Ratcliffe, N. A. and Rowley, A. F. (Eds.), Invertebrate blood cells Vol. 2, Arthropods to urochordates, invertebrate and vertebrates compared, Pergamon Press, London 1981, p. 565.

28 Morris, R. J., Letarte-Muirhead, M. and Williams, A. F., Eur. J. Immunol. 1975. 5: 282.

29 Barclay, A., Letarte-Muirhead, M. and Williams, A. F., Biochem. J. 1975. 151: 699 .

30 Lowry, O. N., Rosebrough, N. J., Farr, A. L. and Randall, R. J., J. Biol. Chem. 1951. 193: 265.

31 Morris, R. J. and Williams, A. F., Eur. J. Immunol. 1975. 5: 274.

32 Greenwood, F. C., Hunter, W. M. and Glover, J. S., Biochem. J. 1963. 89: 114

33 Plesner, T., Norgaard-Pedersen, B. and Boenisch, T., Scand. J. Clin. Lab. Invest. 1975. 35: 729

34 Shalev, A. and Zuckerman, F., Immunology 1984. 50: 667.

35 Durda, P. J. and Gottlieb, P. D., J. Exp. Med. 1976. 144: 476.

36 Cuatrecasas, P., J. Biol. Chem. 1970. 245: 3059.

37 Gahmberg, C. and Hakomori, S. I., Proc. Natl. Acad. Sci. USA 1973. $70: 3329$.

38 Laemmli, U. K., Nature 1970. 227: 680.

39 Barclay, A. N., Letarte-Muirhead, M., Williams, A. F. and Faulkes, A. R., Nature 1976. 263: 563.

40 Reif, A. E. and Allen, J. M. V., J. Exp. Med. 1964. 120: 413.

41 Mason, D. W. and Williams, A. F., Biochem. J. 1980. 187: 1.

42 Zwerner, R. K., Barstad, P. A. and Acton, R. T., J. Exp. Med. 1977. 146: 986.

\author{
Matthias J. Reddehase, \\ James H. Cox and \\ Ulrich H. Koszinowski
}

Federal Research Centre for Virus Diseases of Animals, Tübingen

\section{Frequency analysis of cytolytic $T$ lymphocyte precursors (CTL-P) generated in vivo during lethal rabies infection of mice II. Rabies virus genus specificity of CTL-P}

\begin{abstract}
Cytolytic T lymphocyte precursors (CTL-P) were sensitized in vivo by intraplantar infection of C57BL/6 mice with a lethal dose of rabies virus, strain ERA (ERA). As a result of sensitization CTL-P matured to interleukin-receptive CTL-P (IL-CTL-P) that could be expanded in vitro to Thy $-1^{+}$, Lyt- $2^{+}$CTL clones in the presence of IL without subjection to antigen-driven selection. After infection with ERA, IL-CTL-Pderived CTL lysed fibroblasts infected with rabies virus but not those infected with another rhabdovirus, the vesicular stomatitis virus. These CTL, however, did not discriminate between fibroblasts infected with the serologically closely related laboratory strains of classic rabies virus, ERA and HEP-Flury, and the serologically distinct rabies-related African isolate Mokola. This finding implies that in vivo sensitized IL-CTL-P recognize common genus-specific determinants expressed on cells infected with members of the lyssavirus genus.
\end{abstract}

[ [ 4502]

Correspondence: Ulrich H. Koszinowski, Federal Research Centre for Virus Diseases of Animals, P.O. Box 1149, D-7400 Tübingen 1, FRG

Abbreviations: $\beta$ PL: $\beta$-Propiolactone Con A: Concanavalin A
CTL: Cytolytic T lymphocytes CTL-P: CTL precursors IL: Inter-
leukin IL-CTL-P: IL-receptive CTL-P LD: Limiting dilution LN:
Lymph node(s) PFU: Plaque-forming units VSV: Vesicular
stomatitis virus VSV-Ind: VSV-Indiana strain ERA: Rabies virus,
strain ERA HEP-Flury: Rabies virus, strain High egg passage-Flury

(c) Verlag Chemie GmbH, D-6940 Weinheim, 1984

\section{Introduction}

Worldwide distribution, wide host-range and a relative antigenic uniformity are attributes of classic rabies virus. The laboratory strain ERA (ERA) is a tissue-culture derivative of the canine street virus SAD, isolated from a dog in South Alabama, and HEP-Flury is a chick-embryo adapted rabies virus of canine/human origin. As revealed by the neutralization pattern obtained with a panel of 18 different groups of monoclonal antibodies $(\mathrm{mAb})$ that are reactive with the rabies 
virus glycoprotein (G-protein), both strains differ in some determinants but share most others and are serologically related not only to each other, but also, in varying degrees, to a series of 32 rabies isolates from different geographic and host-species origin [1]. None of these mAb, however, neutralized the African isolates Lagos bat virus (LBV) and Mokola virus (Mokola) which have been classified by Shope $[2,3]$ as "rabies-related". The relation to classic rabies could be confirmed by the reaction pattern of $\mathrm{mAb}$ reactive with the nucleocapsid antigens [1]. Polyclonal rabbit anti-ERA virion antiserum could neutralize all tested isolates and also LBV, but not Mokola. This obvious antigenic distance between ERA and Mokola on the level of the G-protein and the interesting epidemiological aspect that Mokola is geographically restricted to a region in sub-Saharan Africa [2,3] prompted us to analyze whether cytolytic $\mathrm{T}$ lymphocytes (CTL) could detect common antigens on cells infected with either of these two distant members of the lyssavirus genus.

In an attempt to define the antigen that is recognized by rhabdovirus-specific CTL the infection of mice with the serologically distinct vesicular stomatitis virus (VSV) strains VSVIndiana (VSV-Ind) and VSV-New Jersey has previously been used as a model ([4-7], reviewed in [8]). Similar to the observations in the immune response to influenza $A$ viruses, the existence of serotype-specific as well as cross-reactive CTL has been reported for the VSV infection. Studies on the immune response to rabies virus could provide an independent control either to confirm or to challenge the results obtained with VSV. One approach to study the specificity of in vivo primed CTL is the direct testing of polyclonal lymphoid populations or of single effector cells [9] from immunized animals without any in vitro cultivation. Due to the very low primary CTL response to rabies virus these protocols are not feasible [10]. On the other hand, the generation of CTL lines or clones requires repeated in vitro restimulations with antigen which may select for only certain clonotypes and hence this approach includes the risk of losing CTL with interesting specificities. This disadvantage can be avoided when the interleukin (IL)-mediated antigen-free expansion of in vivo sensitized IL-receptive CTL precursors (IL-CTL-P) is used to define the specificity repertoire of CTL [10]. In the present report it is demonstrated that IL-CTL-P generated during infection with rabies virus recognize common determinants expressed on cells infected with serologically distant members of the lyssavirus genus.

\section{Materials and methods}

\subsection{Viruses}

HEP-Flury and the plaque-purified SAD-derived ERA strain of rabies virus were obtained from T. J. Wiktor and VSV-Ind was obtained from R. E. Dierks. Mokola virus, formerly named Ibadan shrew virus IbAn 27377 strain [2, 3], was contributed by $R$. Shope. The viruses were propagated in BHK 21-clone 13 cells [11] and sucrose gradient purified as described [12].

\subsection{In vivo induction of cellular immune responses}

Female C57BL/6 (H-2 ${ }^{\mathrm{b}}$ haplotype) mice, usually used at 6-8 weeks of age, were infected at the left hind footpad with $10^{7}$ plaque-forming units (PFU) of either VSV-Ind or ERA.
Application of this dose of ERA at that site causes death within 6-12 days post-infection. For immunization, ERA was inactivated with $\beta$-propiolactone ( $\beta$ PL; Serva, Heidelberg, FRG) as described [13]. The draining popliteal lymph nodes (LN) were dissected 4 days later and served as source of sensitized lymphocytes.

\subsection{Microculture system and cytolytic assay}

Methods and strategies to determine relative frequencies of rabies-sensitized IL-CTL-P have been described in the preceding report [10]. In brief, graded numbers of in vivo sensitized LN lymphocytes, starting with $1.6 \times 10^{5}$ cells, were cultivated with no addition of antigen in 96-well round-bottomed microtiter plates (Greiner, Nürtingen, FRG) for 6 days in minimum essential medium- $\alpha$ without nucleosides (Gibco Europe, Karlsruhe, FRG) which was supplemented as described [14], plus $10 \%$ fetal calf serum (Seromed, München, FRG) and $20 \%$ IL 2-containing medium. CTL generated in these microcultures were detected in a $3-\mathrm{h}{ }^{51} \mathrm{Cr}$-release assay with $10^{3}$ infected and labeled C57BL/6-derived MC57G fibrosarcoma cells [15] as targets. Infection with the rabies viruses ERA, HEP-Flury and Mokola was performed $24 \mathrm{~h}$ before use at a dose of $10 \mathrm{PFU} / \mathrm{cell}$ in the presence of $50 \mu \mathrm{g}$ DEAE-dextran (Pharmacia, Uppsala, Sweden) per $\mathrm{ml}$ to enhance adsorption and penetration of virus [13]. Infection with VSV-Ind was done at the same dose without DEAE-dextran for $16 \mathrm{~h}$. To test lectin-dependent cytotoxicity, concanavalin A (Con A, Pharmacia) was added at a final dose of $10 \mu \mathrm{g} / \mathrm{ml}$ to noninfected MC57G cells and CTL during the effector phase. This dose was found to be optimal for this type of target cell. Precursor frequencies were estimated from the proportion of nonresponding microcultures by two independent methods, the maximum likelihood (ML, [16]) and the minimum $\chi^{2}$ (MC, [17]) evaluation. According to the proposition of Taswell [17] an estimate was accepted for a probability $p>0.05$ and a ML$\mathrm{MC}$ deviation $<10 \%$. Only the results of the MC evaluation are given in the tables. Calculations were carried out on a Commodore CBM model $4032 / 80$ microcomputer with software written in BASIC.

\section{Results}

\subsection{IL-CTL-P are specific for the sensitizing agent}

Viral infection causes the sensitization and expansion of CTL$P$ with specificity for viral or virus-induced antigens. In addition, nonspecific mitogenic effects of the virus could activate precursors with unrelated specificities. In the limiting dilution (LD) experiment shown in Table 1 the frequency of IL-CTL-P was found to be 1 in 39900 lymphocytes when tested on ERAinfected fibroblast targets, whereas lysis of noninfected fibroblasts was not observed. With the intention to detect all ILCTL-P-derived CTL irrespective of their antigen-specificity, the requirement for antigen was circumvented by the addition of the lectin Con A to the effector phase of the cell-mediated lysis assay [18]. The frequency was then found to be $1 \mathrm{IL}-\mathrm{CTL}$ $P$ in 36400 lymphocytes, i.e. not significantly different to the frequency evaluated with ERA-infected fibroblasts as target cells. These results indicate that practically all IL-CTL-P are specific for the sensitizing agent. IL-CTL-P-derived CTL expressed the Thy-1.2 and Lyt-2.2 antigens (not shown) and thus a contribution of natural killer cells [19] can be disregarded in this system. 


\subsection{IL-CTL-P can be induced by infection and by immunization}

To test whether the generation of IL-CTL-P requires sensitization by an immunogen that might be solely presented during in vivo infection, the frequencies of IL-CTL-P were determined after infection as well as after immunization with different

Table 1. After rabies virus infection only antigen-specific IL-CTL-P are generated ${ }^{\text {a) }}$

$\begin{array}{lccc}\text { Target cells } & \begin{array}{c}\text { IL-CTL-P } \\ \text { frequency }\end{array} & \begin{array}{c}\text { Number of } \\ \text { IL-CTL-P } \\ (95 \% \text { confidence } \\ \text { limits })\end{array} & \text { pMC }^{\left.\mathbf{c}^{\mathrm{s}}\right)} \\ \text { ERA-MC57G } & 1 / 39900 & 25(19-32) & 0.32 \\ \text { MC57G } & <1 / 160000^{\mathrm{d})} & 27(21-34) & - \\ \text { Con A + MC57G } & 1 / 36400 & 27\end{array}$

a) LD analysis of 4-day ERA-sensitized LN lymphocytes was performed with 36 replicate microcultures per dilution.

b) Per $10^{6}$ LN lymphocytes.

c) Probability according to minimum $\chi^{2}$ (MC) evaluation.

d) The percentage of nonresponding microcultures exceeded $36.8 \%$ at the highest cell dose under test that was $1.6 \times 10^{5}$ cells/well. doses of $\beta$ PL-inactivated virus. $A$ dose of inactivated virus equivalent to the dose of infectious virus used for lethal infection did not elicit a measurable response (Table 2). This failure, however, could be overcome when the dose was increased by 10 -fold.

\subsection{Discrimination between distinct rhabdovirus serogroups}

C57BL/6 mice were infected either with ERA, or with VSVInd. Table 3 documents that ERA-immune CTL lysed fibroblasts which were infected with ERA but not those infected with VSV-Ind. Accordingly, VSV-Ind-immune CTL lysed only VSV-Ind-infected fibroblasts and not those infected with ERA. These findings allow the conclusion that cells infected with either of these two rhabdoviruses do not share antigens which are relevant for recognition by CTL.

\subsection{Cells infected with subtypes of rabies virus share an antigen}

The main objective of this study was to test whether CTL that were derived by antigen-independent in vitro expansion of in vivo activated IL-CTL-P [10] could detect antigenic relationships between serologically distant members of the rabies virus group as was the case for VSV-specific CTL after repeated

Figure 1. ERA-induced CTL recognize cells infected with the distantly related Mokola virus. CTL cultures in 72 replicates were obtained after a 6-day IL-mediated expansion of $1.6 \times 10^{5}$ 4-day ERAsensitized LN lymphocytes and were split into 6 identical, corresponding fractions. Two of these fractions were assayed on ERA-infected MC57G cells (comparison $\mathrm{A} \rightarrow \mathrm{B}$, vertically hatched areas), one fraction for control on noninfected MC57G cells and the remaining fractions on MC57G cells infected with either HEP, Mokola or VSV-Ind. Lytic activity was neither observed on the noninfected target (not shown) nor on the VSV-Ind-infected target (comparison $\mathrm{A} \rightarrow \mathrm{C}$, horizontally hatched area in the left part of the figure) and the activity distribution measured with HEP-MC57G closely resembled that observed with Mokola-MC57G (Mokola depicted only; comparison $\mathrm{A} \rightarrow \mathrm{C}$, horizontally hatched area in the right part of the figure). For the sake of clarity, the lytic activities are not depicted as individual points, but instead, the distributions are given as hatched areas. The dashed lines indicate the minimum positive detection levels for the target ERA-MC57G that range from 4.7 to $5.4 \%$ specific lysis. The corresponding level is $2.2 \%$ for Mokola-MC57G and $4.0 \%$ for VSV-Ind-MC57G.

Table 2. Immune response to infectious and inactivated virus

\begin{tabular}{|c|c|c|c|c|}
\hline Priming antigen ${ }^{a)}$ & $\begin{array}{l}\text { Cells/LN } \\
\times 10^{-6^{6}}\end{array}$ & $\begin{array}{l}\text { IL-CTL-P } \\
\text { frequency }\end{array}$ & $\begin{array}{c}\text { Number of } \\
\text { IL-CTL-P) } \\
\text { (95\% } \\
\text { confidence } \\
\text { limits) }\end{array}$ & $\mathrm{pMC}$ \\
\hline $\begin{array}{ll}10^{7} \text { PFU } & \text { ERA infectious } \\
10^{7} \text { PFU-E } & \text { ERA- } \beta P L \\
10^{8} \text { PFU-E } & \text { ERA- } \beta P L\end{array}$ & $\begin{array}{l}5.9 \\
1.2 \\
5.3\end{array}$ & $\begin{array}{r}1 / 18100 \\
<1 / 160000 \\
1 / 36400\end{array}$ & $55(42-69)$ & $\begin{array}{c}0.31 \\
0.08\end{array}$ \\
\hline
\end{tabular}

a) Mice were either infected with $10^{7} \mathrm{PFU}$ infectious rabies virus, strain ERA, or primed with $\beta$ PL-inactivated virions at a dose equivalent to $10^{7}$ or $10^{8} \mathrm{PFU}$, respectively. LD analysis of 4-day sensitized LN lymphocytes was performed with 36 replicate microcultures per dilution. ERA-infected MC57G fibroblasts were used as target cells. In neither of the priming protocols could significant lytic activity be detected on noninfected MC57G targets.

b) Average number of lymphocytes per draining popliteal LN estimated from a pool of 10 animals per group.

c) Per $10^{6} \mathrm{LN}$ lymphocytes.

d) $P F U-E=$ Plaque-forming units equivalent. 
Table 3. Rhabdovirus-immune CTL discriminate between different serogroups ${ }^{\text {a) }}$

Target cell $\quad$ ERA priming
Lytic activity (range)

$\begin{array}{lrr}\text { ERA-MC57G } & 45.9(27.2-77.2) & 1.4(-2.1-17.3) \\ \text { VSV-Ind-MC57G } & 0.6(-1.9-5.2) & 62.2(51.7-75.2)\end{array}$

a) Mice were infected with $10^{7}$ PFU of either rabies virus, strain ERA, or VSV-Ind and $1.6 \times 10^{5} 4$-day sensitized LN lymphocytes per microculture in 72 replicates were expanded for 6 days in the presence of IL with no antigen. Thereafter the CTL-containing cultures were split twice and the aliquots tested on the target cells indicated.

b) Lytic activity is given as median value of 72 replicates, expressed in $\%$ specific lysis.

restimulations [7]. Rabies virus-specific CTL were obtained by infection of C57BL/6 mice with ERA and subsequent in vitro propagation of IL-CTL-P in the presence of IL only. Individual cultures were then split 6-fold and tested on fibroblast target cells which were either noninfected or infected with the sensitizing strain ERA, the closely related strain HEP-Flury, the distantly related strain Mokola and the nonrelated rhabdovirus VSV-Ind. The results are depicted in Fig. 1. Again, noninfected target cells as well as VSV-Ind-infected target cells were not lysed by ERA-immune CTL but, in addition to ERA-infected target cells, HEP-Flury-infected target cells and also those infected with Mokola virus were lysed.

These results pointed to the presence of a common antigen expressed on cells infected with members of the lyssavirus genus. The observation that the lytic activity detected on ERA-infected fibroblasts exceeded that detected on Mokolainfected (Fig. 1) as well as on HEP-Flury-infected (not shown in Fig. 1) fibroblasts could suggest the existence of ERA serotype-specific CTL in addition to the genus-specific CTL. Differences in the lysis, however, may either be due to separate effector populations specific for distinct antigens or may reflect differences in the susceptibility of the target cells to lysis by one effector population due to quantitative differences in the expression of one type of antigen. Only the rank order of the lytic activities detected in individual cultures by splitting and testing on a panel of target cells is independent of the amount of antigen and, therefore, rank correlation analysis [20] is an appropriate method to decide between these alternatives. The lytic activities measured on the three types of rabiesinfected target cells were found to be strictly correlated (probability for independent distribution $\leqq 0.001$ in all combinations) and thus it can be concluded that practically all CTL are genus specific. Attempts to inhibit these ERA-induced rabies virus genus-specific CTL with polyclonal rabbit antisera to the ERA virion, G-protein, NC-protein or M2-protein failed (data not shown).

\section{Discussion}

This report is part of a study on the CTL response of mice during lethal infection with rabies virus. In a preceding report [10] it has been demonstrated that in vivo sensitization of rabies-specific CTL-P and in vitro propagation of these activated CTL-P can be separated. Based on this prerequisite a
LD protocol was established that allowed us to evaluate the number of activated CTL-P present during early stages of viral infection. Different frequencies, considered to indicate the presence of different maturation stages of triggered CTL-P, were obtained depending on whether the sensitized CTL-P were only expanded by IL (IL-CTL-P) or were subjected to a second sensitization by antigen (antigen-dependent CTL-P). The distinction of cells on the basis of maturity has also been demonstrated in a herpes virus system with quite different pathogenesis [14] and therefore reflects a more common phenomenon in the in vivo maturation of antiviral CTL.

The generation of CTL by expansion of IL-CTL-P is not influenced by selective effects of antigen in vitro and this advantage was used in the present report to define the fine specificity of anti-rabies CTL. Our study allows the following conclusions: first, practically all IL-CTL-P are specific for the sensitizing agent and belong to the lineage of Thy $-1^{+}$, Lyt- $2^{+}$lymphocytes. This finding implies that rabies virus does not exert an unspecific mitogenic effect. Second, activation of IL-CTL-P is more pronounced during infection than after vaccination with inactivated virus, provided equivalent doses are used. In earlier reports on the CTL response to rabies virus $[21,22]$, appropriate doses of inactivated virus were shown to be highly immunogenic and it was stated that only attenuated viruses, but not virulent strains that cause lethal disease, could elicit a CTL response. Our findings after infection with a lethal dose of an attenuated virus add the information that a fatal outcome of the infection within the short period of 6-12 days does not exclude the generation of a CTL response. As far as it is known, there is no extensive rabies virus multiplication at the site of subcutaneous or intramuscular injection [23]. Yet, a more efficient presentation of the antigen or expression of qualitatively different antigens in vivo may be discussed as explanations for the high immunogenicity of infectious virus. Third, practically all rabies-immune IL-CTL-P and their progeny-CTL are "cross-reactive" for cells infected with serologically distant members of the same genus, whereas cells infected with a member of a different genus, e.g. VSV-Ind, are not recognized. Since the specificity of CTL obviously reflects a relatedness that is also confirmed by independent virological criteria, the so-called "cross-reactivity" is most likely due to recognition of common "genus-specific" determinants.

Recently it has been reported that all long-term cloned CTL lines derived from VSV-Ind-sensitized CTL do not discriminate between targets infected with the serologically distinct VSV strains VSV-Ind and VSV-New Jersey [7]. To establish such clones, repeated in vitro restimulations with antigen were necessary. The observations after ERA infection of mice confirm this finding in an independent rhabdovirus system and, more important, by the use of an experimental protocol that intentionally avoids in vitro restimulation. Hence, it has to be concluded that the predominance of genus-specific CTL indeed reflects the in vivo situation after infection and does not result from in vitro selection by antigen.

We thank Ms. Waltraud Umrath and Monika Schwarz for expert technical assistance and Mr. Michael Reddehase for designing the computer software.

Received March 29, 1984; in revised form June 25, 1984. 


\section{References}

1 Schneider, L. G. and Meyer, S., in Bishop, D. H. L. and Compans, R. W. (Eds.), The Replication of Negative Strand Viruses, Elsevier/North-Holland, Amsterdam 1981, p. 947.

2 Shope, R. E., Murphy, F. A., Harrison, A. K., Causey, O. R., Kemp, G. E., Simpson, D. I. H. and Moore, D. L., J. Virol. 1970. 6: 690 .

3 Shope, R., in Baer, G. M. (Ed.), The Natural History of Rabies, Vol. I, Academic Press, New York 1975, p. 141.

4 Zinkernagel, R. M., Althage, A. and Holland, J., J. Immunol. 1978. 121: 744 .

5 Rosenthal, K. L. and Zinkernagel, R. M., J. Immunol. 1980. 124: 2301.

6 Sethi, K. K. and Brandis, H., Eur. J. Immunol. 1980. 10: 268.

7 Rosenthal, K. L., Oldstone, M. B. A., Hengartner, H. and Zinkernagel, R. M., J. Immunol. 1983. 131: 475.

8 Zinkernagel, R. M. and Rosenthal, K. L., Immunol. Rev. 1981. 58: 131 .

9 Grimm, E. A. and Bonavida, B., J. Immunol. 1979. 123: 2861.

10 Reddehase, M. J., Cox, J. H. and Koszinowski, U. H., Eur. J. Immunol. 1982. 12: 519.
11 Stoker, M. and McPherson, J., Nature 1964. 203: 1355.

12 Cox, J. H., Dietzschold, B. and Schneider, L. G., Infect. Immun. 1977. 16: 754.

13 Koprowski, H., in Kaplan, M. M. and Koprowski, H. (Eds.), Laboratory Techniques in Rabies, World Health Organization, Geneva 1973 , p. 256.

14 Reddehase, M. J., Keil, G. M. and Koszinowski, U. H., J. Immunol. 1984. 132: 482.

15 Aden, D. P. and Knowles, B. B., Immunogenetics 1976. 3: 209.

16 Fazekas de St. Groth, S., J. Immunol. Methods 1982. 49: R11.

17 Taswell, C., J. Immunol. 1981. 126: 1614.

18 Berke, G., Immunol. Rev. 1983. $72: 5$.

19 Minato, N., Reid, L. and Bloom, B. R., J. Exp. Med. 1981. 154: 750.

20 Reddehase, M. J., Keil, G. M. and Koszinowski, U. H., Eur. J. Immunol. 1984. 14: 56.

21 Wiktor, T. J., Dev. Biol. Stand. 1978. 40:255.

22 Wiktor, T. J., Doherty, P. C. and Koprowski, H., Proc. Natl. Acad. Sci. USA 1977. 74: 334.

23 Baer, G. M., in Baer, G. M. (Ed.), The Natural History of Rabies, Vol. I, Academic Press, New York 1975, p. 181.

\section{Claudia Berek}

Medical Research Council, Laboratory of Molecular Biology, Cambridge

\section{The $\mathrm{D}$ segment defines the $\mathrm{T} 15$ idiotype: the immunoresponse of $\mathrm{A} / \mathrm{J}$ mice to Pneumococcus pneumoniae* $^{*}$}

In the immune response of BALB/c mice $\left(\operatorname{Igh}^{a}\right)$ to Pneumococcus the majority of antibodies express the idiotype of the myeloma protein TEPC15 (T15). In contrast mice of the $\mathrm{A} / \mathrm{J}$ strain $\left(\operatorname{Igh}^{\mathrm{C}}\right)$ do not express this idiotype. Using $(\mathrm{BALB} / \mathrm{c} \times \mathrm{A} / \mathrm{J}) \mathrm{F}_{1}, \mathrm{~F}_{2}$ or backcross mice it could be shown that in allotype heterozygous animals $\left(\mathrm{Igh}^{\mathrm{a} / \mathrm{e}}\right.$ ) Pneumococcus pneumoniae preferentially stimulates B cells expressing a heavy chain (H) encoded by genes in the $\mathrm{BALB} / \mathrm{c} \mathrm{H}$ chain gene complex. Phosphorylcholine (PC)specific hybridoma lines were established from BALB/c and $\mathrm{A} / \mathrm{J}$ spleen cells and idiotypically analyzed using monoclonal antibodies $(\mathrm{mAb})$ specific for the T15 idiotopes $32 / 65,10 / 13,16 / 13$ or $21 \mathrm{~A} 5$. Whereas the majority of the BALB/c PCbinding $\mathrm{mAb}$ express these idiotopes, only some of the $\mathrm{A} / \mathrm{J} \mathrm{mAb}$ are positive for one or the other of the idiotopes formed by the variable (V) regions of the $\mathrm{H}$ and the light chain of the myeloma protein T15. However, $80 \%$ of the A/J PC-binding hybridoma proteins were bound by the anti-idiotopic mAb 21A5. This $\mathrm{mAb}$ is specific for a determinant partially formed by the $C_{\alpha}$ and partially by the $V$ regions of the myeloma protein T15. The mRNA of one of these $\mathrm{T}^{-} \mathrm{A} / \mathrm{J}$ PC-binding hybridoma lines was sequenced. $V_{H}$ and $V_{x}$ were identical with sequences found for BALB/c T15-like antibodies. The sequence of the $\mathrm{D}$ segment was structurally very different. The importance of the D segment in the dominant expression of the T15 idiotype is discussed.

\section{[I 4578] 1 Introduction}

* Part of the work was carried out at the Basel Institute of Immunology, founded and supported by F. Hoffmann-La Roche and Co.

Correspondence: Claudia Berek, Medical Research Council, Laboratory of Molecular Biology, Hills Road, Cambridge, GB

Abbreviations: C: Constant region H: Heavy (chains) L: Light (chains) PC: Phosphorylcholine PN: Pneumococcus pneumonia SRBC: Sheep red blood cells T15: TEPC 15 V: Variable region mAb: Monoclonal antibody(ies) PFC: Plaque-forming cells
In an immune response to phosphorylcholine (PC) three types of antibodies are made, which are characterized by their structural resemblance to the myeloma proteins TEPC 15 (T15), MOPC 167 (M167) and McPC603 (M603). They all have the same type of heavy $(\mathrm{H})$ chain which combines with $x$ light $(\mathrm{L})$ chains of the $V_{x 22}, V_{x 24}$ or $V_{x 8}$ subgroups, respectively [1]. The $\mathrm{H}$ chain of the $\mathrm{PC}$-binding antibodies is encoded by a single gene of the $T 15 \mathrm{~V}_{\mathrm{H}}$ gene family, the $\mathrm{V} 1$ gene [2]. Screening DNA in a Southern blot analysis with a T15 probe indicated 